Draft version August 27, 2021

Preprint typeset using $\mathrm{LTT}_{\mathrm{E}} \mathrm{X}$ style emulateapj v. 08/22/09

\title{
CAN WE REPRODUCE THE X-RAY BACKGROUND SPECTRAL SHAPE USING LOCAL AGN?
}

\author{
Ranjan V. Vasudevan ${ }^{1, *}$, Richard F. Mushotzky ${ }^{1}$, Poshak Gandhi $^{2},{ }^{3}$ \\ Draft version August 27, 2021
}

\begin{abstract}
The X-ray background (XRB) is due to the aggregate of active galactic nuclei (AGN), which peak in activity at $z \sim 1$ and is often modeled as the sum of different proportions of unabsorbed, moderatelyand heavily-absorbed AGN. We present the summed spectrum of a complete sample of local AGN (the Northern Galactic Cap of the 58-month Swift/BAT catalog, $z<0.2$ ) using $0.4-200 \mathrm{keV}$ data and directly determine the different proportions of unabsorbed, moderately and heavily-absorbed AGN that make up the summed spectrum. This stacked low redshift AGN spectrum is remarkably similar in shape to the XRB spectrum (when shifted to $z \sim 1$ ), but the observed proportions of different absorption populations differ from most XRB synthesis models. AGN with Compton-thick absorption account for only $\sim 12 \%$ of the sample, but produce a significant contribution to the overall spectrum. We confirm that Compton reflection is more prominent in moderately-absorbed AGN and that the photon index differs intrinsically between unabsorbed and absorbed AGN. The AGN in our sample account for only $\sim 1 \%$ of the XRB intensity. The reproduction of the XRB spectral shape suggests that strong evolution in individual AGN properties is not required between $z \sim 0$ and 1 .

Subject headings:
\end{abstract}

\section{INTRODUCTION}

The X-ray background (XRB) has been extensively studied from $1-500 \mathrm{keV}$ and is the combined emission from AGN with varying degrees of obscuration and Compton reflection modifying the spectral shape of each object (e.g., Treister et al. 2009, Gilli et al. 2007 - T09 and G07 respectively, hereafter). The excellent angular resolution and low background of Chandra have allowed an analysis of the properties of the individual AGN that make up most of the 1-6 keV XRB (Worslev et al. 2006). However, these objects lie at redshifts $\langle z\rangle \sim 1.0$ (e.g., Soltan 2008), and therefore the spectral quality is poor in comparison to nearby AGN. Additionally, spectral data are typically only available in the Chandra/XMM band (observed frame $<10 \mathrm{keV}$ ) and do not extend to 15 $40 \mathrm{keV}$, where the majority of the XRB power lies. It was therefore not possible to uniquely reconstruct the XRB spectrum across $1-500 \mathrm{keV}$ by combining the emission from individual AGN.

Numerous studies predict the different populations of AGN required to reproduce the XRB spectral shape, by simulating the observed XRB as the sum of different proportions of unabsorbed, absorbed Comptonthin and absorbed Compton-thick spectra, (G07, T09, Akylas et al. 2012). The fraction of Compton-thick sources remains uncertain as does the distribution of Compton reflection strengths and high energy cut-offs. Recently Akylas et al. (2012) showed that a wide range of these parameters can fit the observed XRB spectrum and redshift distribution of sources. Without stricter

\footnotetext{
${ }^{1}$ Department of Astronomy, University of Maryland, College Park, MD, 20742

2 Institute of Space and Astronautical Science, Japan Aerospace Exploration Agency, 3-1-1 Yoshinodai, chuo-ku, Sagamihara, Kanagawa 252-5210, Japan

${ }^{3}$ Department of Physics, Durham University, South Road, Durham DH1 3LE, UK

*ranjan@astro.umd.edu
}

constraints on the source properties, there remains a large uncertainty in the models. We adopt a 'bottom-up' approach to understanding the make-up of the XRB by examining the composite spectra of local AGN over 0.5$200 \mathrm{keV}$. This approach was pioneered by Sazonov et al. (2008) with a very thorough analysis of INTEGRAL and RXTE data (3-300 keV), and developed further by Ricci et al. (2011) (10-300 keV). We utilize data from ASCA, Swift/XRT, XMM and Swift/BAT, and compare the composite with the XRB spectrum and synthesis models. We show that the sum of the local sources provides an excellent match to the observed XRB shifted to $z \sim 0$ indicating that despite expected evolution in AGN population properties (due to observed changes in luminosity, accretion rate, host galaxy type, obscured AGN fraction between $z=1$ and now, e.g. Barger et al. 2005; Aird et al. 2012; Delgado-Serrano et al. 2010), the overall combined emission from such sources stays the same.

\section{SAMPLE}

The 58-month Swift Burst Alert Telescope (BAT) allsky, hard-X-ray-selected survey of local $\mathrm{AGN}^{5}(\langle z\rangle=$ $0.043)$ is complete and relatively unbiased to absorption, thanks to the efficient hard X-ray selection. We employ the detailed X-ray analysis of our companion paper Vasudevan et al. (2013) (V13 hereafter) for a complete subsample from the 58-month catalog, the Northern Galactic Cap (all radio-quiet sources with Galactic latitude $b>50^{\circ}$, flux limit $4 \times 10^{-12} \mathrm{erg} \mathrm{s}^{-1} \mathrm{~cm}^{-2}$ ). In V13, 0.4-10 keV data from XMM, Swift/XRT and ASCA, along with Swift/BAT data (14-200 keV), are fitted to produce estimates of the column density, spectral slope and reflection fraction $(R)$ for each object.

\section{GENERATING THE STACKED SPECTRUM OF THE BAT AGN}

${ }^{5}$ http://heasarc.gsfc.nasa.gov/docs/swift/results/bs58mon/ 
The V13 study provides a best-fitting model for each source. The data in the $0.4-10 \mathrm{keV}$ band are from different satellites and of variable quality, so we use the best-fit models to parameterize their spectra. For each object, we extract the model using 1000 bins over $0.4-$ $10 \mathrm{keV}$ (using the 'dummyrsp' command in XSPEC) providing a spectrum for each object over a common energy grid. We sum flux densities over this grid to generate the summed spectrum. We perform this for the whole sample (95 objects with good spectra) and for different absorption regimes: unabsorbed $\left(\log \mathrm{N}_{\mathrm{H}}<21,27\right.$ objects), absorbed Compton-thin $\left(21<\log \mathrm{N}_{\mathrm{H}}<24,51\right.$ objects) and absorbed Compton-thick $\left(\log \mathrm{N}_{\mathrm{H}}>24,12\right.$ objects), using the same $\log \mathrm{N}_{\mathrm{H}}$ bins as G07 for comparison with their results.

For thirteen objects, their $N_{\mathrm{H}}$ values are uncertain due to poorer quality $0.4-10 \mathrm{keV}$ data. We therefore produce two sets of summed spectra; one in which these objects take their minimal $N_{\mathrm{H}}$ values, and one in which they take their maximal $N_{\mathrm{H}}$ values.

Above $10 \mathrm{keV}$, the eight energy channels of BAT (at fixed energies between $14-200 \mathrm{keV}$ ), already provide a common energy grid over which to sum the fluxes. We generate the summed BAT spectra as above, for the whole sample and for different absorption regimes. For comparison with previous studies, we convert the stacked spectra to $E F_{E}$ format (Fig. 1).

The BAT AGN show significant variability, so if the 0.4-10 keV data for a particular source was taken during the time-frame of the BAT survey, V13 renormalized the BAT spectrum based on the source flux in the BAT band at the observation time of the XMM/XRT data, using these renormalized spectra to determine $N_{\mathrm{H}}$. For this paper we use the uncorrected (non-renormalized) BAT spectra providing a consistent approach for all sources, since renormalization was only possible for $40 \%$ of objects. The summed emission should anyhow be relatively independent of intra-source variability.

The stacked model fits below $10 \mathrm{keV}$ do not have error estimates on the summed fluxes in each energy bin, but above $10 \mathrm{keV}$, for each energy channel we add the errors from each AGN in quadrature and take the square root of the sum to determine the total error. The total summed BAT spectrum has very small formal errors, but some of the $N_{\mathrm{H}}$ selected subsets have larger errors, particularly Compton-thick sources, because of the small number of such sources (12). Thus the Compton-thick fraction remains a key uncertainty in generating the total AGN spectrum, even when using a 'complete' AGN sample. We caution that even hard X-ray selected catalogs are somewhat biased against Compton-thick AGN (Malizia et al. 2009; Burlon et al. 2011, V13).

The highest energy BAT channel has relatively large errors for all $N_{\mathrm{H}}$ groups perhaps indicative of a calibration problem in this channel; other studies (e.g., Burlon et al. 2011) find similar anomalies. Our analysis is therefore most reliable below $\sim 150 \mathrm{keV}$, still covering the peak of the XRB emission, critical for determining the total AGN power budget.

We overplot the XRB spectrum from G07 (a composite from many missions), scaled down in flux to match the summed emission from the BAT sources in $2-200 \mathrm{keV}$. We employ the XRB spectral shape from HEAO-1 (Boldt 1987) to determine the $2-200 \mathrm{keV}$ 'average' rest-frame
XRB intensity (i.e. $1-100 \mathrm{keV}$ for the observed XRB). Once correcting for our survey area (1.47 steradians), our stacked spectrum normalisation represents $1.2 \%$ of the XRB flux (i.e. 82 times lower in flux). We crosscheck this against the more recent measurement of the XRB using Swift/XRT and BAT by Moretti et al. (2009) and find very similar results (our BAT sources constitute $\sim 1 \%$ of their XRB intensity). Other determinations of the XRB using BeppoSAX (Frontera et al. 2007) and INTEGRAL (Churazov et al. 2007; Türler et al. 2010) yield different overall normalizations, but the comparison between these and many other XRB measurements in Ajello et al. (2008) (Figures 13 and 14) show consistency within errors, and we are mainly concerned with the spectral shape rather than the normalization in this study. We also shift the XRB spectrum into the 'average' rest-frame, assuming an average redshift of XRB sources of $\sim 1.0$. We also apply the normalization and redshift scalings to the XRB synthesis model components (for different $N_{\mathrm{H}}$ bins) in the G07 model.

\section{RESULTS}

The peak in the summed BAT spectrum coincides with that from the XRB at $\sim 60 \mathrm{keV}$ (corresponding to $\sim 30 \mathrm{keV}$ for $z \sim 1 \mathrm{AGN}$ ). If the $0.4-200 \mathrm{keV}$ spectral shape of AGN at $z \sim 0$ is representative of the AGN spectral shape throughout AGN history, this provides an elegant confirmation that most of the accretion contributing to the XRB occurs at $z \sim 1$. The normalization of the $>10 \mathrm{keV}$ stacked spectrum lies above the scaled XRB emission; but the small number of sources (95) yields an intrinsic $10 \%$ statistical fluctuation in our normalization, as does the uncertainty in the XRB normalization; the results are therefore consistent within errors. We test that our stacked spectrum is not dominated by a few bright sources by checking the effect of omitting the brightest three; the $>10 \mathrm{keV}$ spectral shape remains unchanged whereas the $<10 \mathrm{keV}$ spectral shape becomes moderately softer within the error range of the measured XRB slope.

The spectral evolution of AGN above $10 \mathrm{keV}$ (restframe) is not known, but a key result of our study is that the combined emission from local AGN is consistent with the (redshift-corrected) XRB shape. Below $10 \mathrm{keV}$, the contribution from unobscured AGN lies a factor of $\sim 1.4$ lower than the G07 model synthesis prediction while that from obscured Compton-thin AGN agrees well with the synthesis model. Above $10 \mathrm{keV}$, the unobscured AGN contribution agrees reasonably well; however the obscured Compton-thin AGN contribution lies 78\% higher but with a similar shape to the G07 model.

We also compare our results with the T09 XRB synthesis model in Fig. 2, splitting obscured and unobscured sources at $\log \mathrm{N}_{\mathrm{H}}=22$ instead, as done by those authors. For down-scaling the XRB shape and model components, here we employ the more recent Swift XRB measurement by Moretti et al. (2009), which has less overall uncertainty in the normalization. Our unobscured and Compton-thick spectra show good agreement, but the obscured class shows a higher contribution both below and above $10 \mathrm{keV}$, with an excess above $10 \mathrm{keV}$ consistent with stronger reflection, perhaps indicating that Compton reflection in Compton thin sources is more im- 

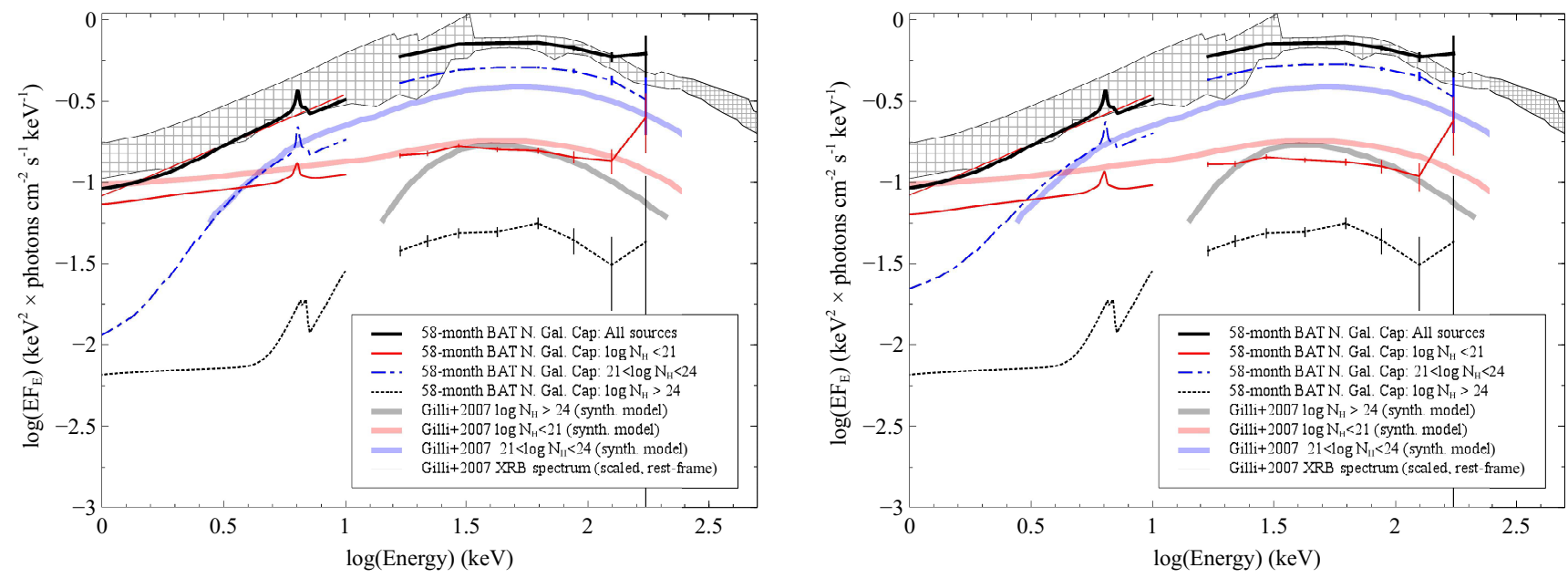

FIG. 1. - Stacked 0.4-200 keV spectrum for the sources in our sample, comparing with G07 XRB synthesis model. Left panel: ambiguous $N_{\mathrm{H}}$ sources take their minimal $N_{\mathrm{H}}$. Right panel: such sources have their maximal $N_{\mathrm{H}}$. See inset key for details.

portant than previously assumed.

The Compton-thick contribution above $10 \mathrm{keV}$ lies below the predictions from the G07 model and is consistent with the T09 model; the higher hard X-ray excess in Compton-thin obscured sources requires less of a Compton-thick component to produce the overall XRB shape.

Akylas et al. (2012) find that reflection at the level of $0.5<R<2$ can be combined with the observed Compton-thick fraction from Swift studies (e.g. Burlon et al. 2011) to explain the XRB. The offset seen here may be too great to be from simple reflection geometries and might require strong light bending for a substantial fraction of sources (e.g. Gandhi et al. 2007). Such sources represent over $50 \%$ of the sample, at the upper limit of the proportion of light-bent sources predicted Gandhi et al. (2007) scenario. A substantial fraction of the individual sources (V13 and Tatum et al. 2013) show such a 'hard excess' consistent with either a higher than expected reflection or an additional highly absorbed spectral component; the other absorption groups do not show such an excess.

\subsection{Parameterising the hard excess using reflection}

Ricci et al. (2011) analyze the INTEGRAL spectra (>10 keV) of 165 hard X-ray selected AGN and perform a comprehensive and complementary analysis. They find that moderately obscured AGN ('MOB', $23<\log \mathrm{N}_{\mathrm{H}}<$ 24) have more pronounced reflection $\left(R=2.2_{-1.1}^{+4.5}\right)$ than unabsorbed $\left(\log \mathrm{N}_{\mathrm{H}}<22\right)$ and lightly-obscured AGN ('LOB', $22<\log \mathrm{N}_{\mathrm{H}}<23$ ).

We split our sample into their categories (39 unabsorbed, $18 \mathrm{LOB}, 25 \mathrm{MOB}, 12$ Compton-thick, assuming minimal $N_{\mathrm{H}}$ when two are available). For the averaged model fits $<10 \mathrm{keV}$, we sample the spectra with 20 bins below $10 \mathrm{keV}$, assume a conservative value of $10 \%$ errors on the flux density in each bin (if the data themselves were stacked, errors on the total spectrum would be much smaller; we estimate errors on the Compton-thick group to be $6 \%$, which should represent the worst case). We fit the $1-200 \mathrm{keV}$ data with the reflection model PEXRAV, assuming defaults for all parameters, allowing the photon index $\Gamma$ and reflection fraction $R$ to vary (we freeze $E_{\mathrm{fold}}$ at $300 \mathrm{keV}$ consistent with the average for BAT AGN, see V13). We assume the same normalization below and above $10 \mathrm{keV}$, since intra-object variability should be smoothed out in a stacked spectrum, and any statistically significant difference in normalization between soft and hard bands should therefore be real. We include a partial covering absorber for sources with $\log \mathrm{N}_{\mathrm{H}}>22$ (model PCFABS(PEXRAV)), and find that the $N_{\mathrm{H}}$ value obtained from the stacked spectra fits are well within the $N_{\mathrm{H}}$ limits of each bin.

Our results (Fig. 3) are in good agreement with Ricci et al. (2011), finding a pronounced reflection component for MOB AGN $\left(\mathrm{R}=2.0_{-0.5}^{+0.6}\right)$. One possible explanation (Ricci et al. 2011) is that for moderately-obscured sources, reflection from Compton-thick clumpy clouds of absorbing material boosts the total reflected flux. The PEXrAV model produces a worse fit to MOB AGN $\left(\chi^{2} /\right.$ d.o.f. $\approx 8$ compared to $\sim 1$ for other classes $)$ overpredicting the $5-10 \mathrm{keV}$ emission, which can be partially resolved by allowing for sub-solar abundances in the PEXRAV fit; however it is unclear why MOB AGN should have intrinsically different abundances. The contours show an evolution towards higher reflection fraction with higher absorption. We assumed a constant inclination angle; in the standard Unified Model (Antonucci 1993) one may expect more edge-on tori for more heavilyabsorbed sources. We investigated this effect along the same lines as Ricci et al. (2011) and find that if inclination increases with absorption, the reflection fraction increases even more strongly with absorption than for a constant angle, indicating a salient difference in the reflection geometry. The reflection fraction for Comptonthick AGN is more uncertain and requires more sophisticated modeling, so we omit it from Fig. 3 ,

The difference of photon indices between absorbed and unabsorbed sources $(\Gamma=1.86 \pm 0.05$ for unabsorbed and $\Gamma=1.76 \pm 0.03$ for MOB AGN) is very similar to that seen by Lanzuisi et al. (2013) for the COSMOS field $(\Gamma=$ $1.89 \pm 0.02$ for Seyfert $1 \mathrm{~s}$ and $\Gamma=1.76 \pm 0.03$ for Seyfert 

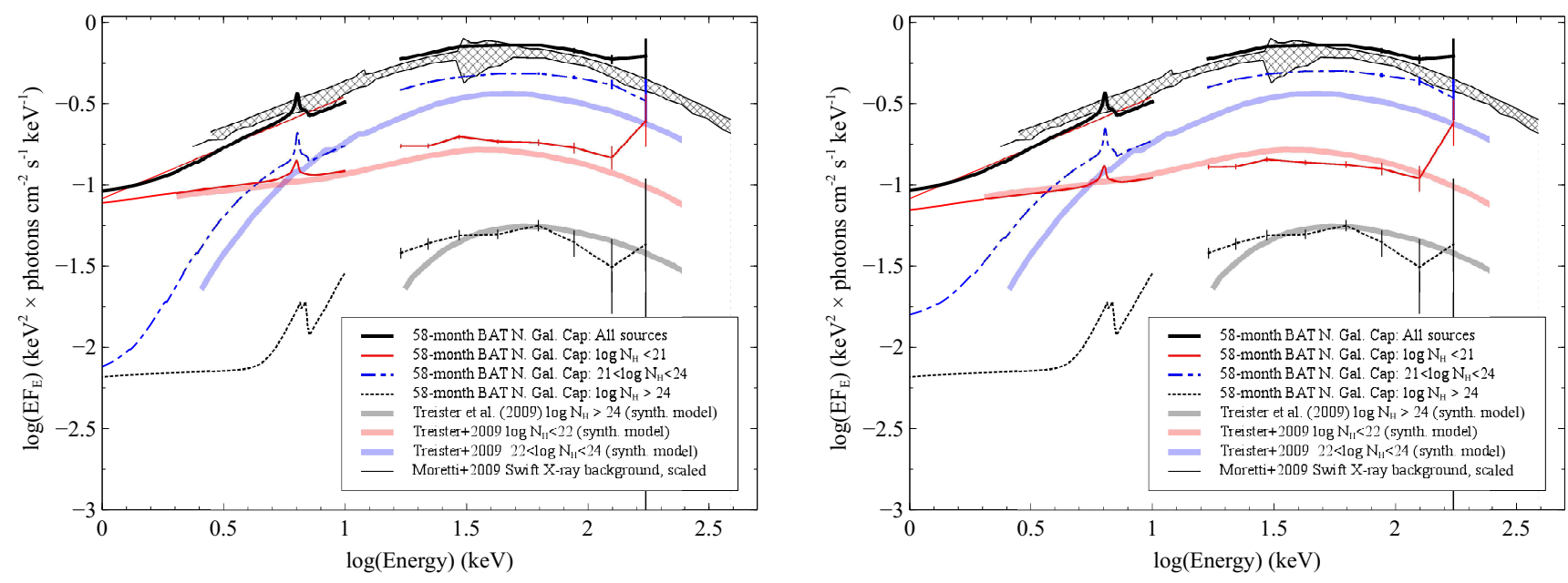

FIG. 2.- Comparison with the T09 XRB synthesis model. Key as in Fig. 1 except the boundary between unabsorbed and absorbed groups is now $\log \mathrm{N}_{\mathrm{H}}=22$, as employed by T09.

$2 \mathrm{~s})$. In the context of a Compton-scattering model for the X-ray continuum, it may indicate that the optical depth 'seen' by accretion disk photons emerging from the Comptonizing corona varies depending on the angle at which the corona is viewed. This indicates some degree of deviation from spherical geometry for the corona, with implications for AGN unification schemes.

One can potentially also account for these results if luminosity is the key driver: since lower luminosity sources have a greater Iron $\mathrm{K} \alpha$ linewidth (Iwasawa \& Taniguchi 1993) and thus an implied higher reflection (Nandra et al. 1997, Piconcelli et al.|2005), the observed changes in reflection with absorption can be ascribed to a luminosity effect rather than geometry. However, stacking the unabsorbed AGN in four different luminosity bins with equal numbers of objects per bin (Fig. 4), shows no trend for luminosity-dependent reflection (for $\log \mathrm{L}_{2-10 \mathrm{keV}}<44.4$ ). This remains a debated issue: such a trend has been observed to varying degrees in smaller samples of high-luminosity quasars (Williams et al. 1992; Page et al. 2005), but our sample does not extend to such luminosities to test this.

\subsection{Objects without 0.1-10 keV spectra and their contribution above $10 \mathrm{keV}$}

Five objects in V13 have poor quality $0.4-10 \mathrm{keV}$ data, precluding accurate determinations of $N_{\mathrm{H}}$; they have not been included in the total stacked spectrum. Since these sources contribute only $\sim 3 \%$ to the total emission at $30 \mathrm{keV}$, their net effect is small (Fig. 5). In the extreme case, if they are all Compton-thick, the percentage of Compton-thick sources could be as high as $\sim 18 \%$.

\section{SUMMARY}

We present the summed $0.4-200 \mathrm{keV}$ spectrum of the AGN in Northern Galactic Cap $\left(b>50^{\circ}\right)$ of the 58month BAT catalog, and compare the observed contribution of AGN with different absorptions to predictions from XRB synthesis models. There is a striking similarity between our stacked spectrum and the XRB spectral shape. Despite the known evolution in the luminosity

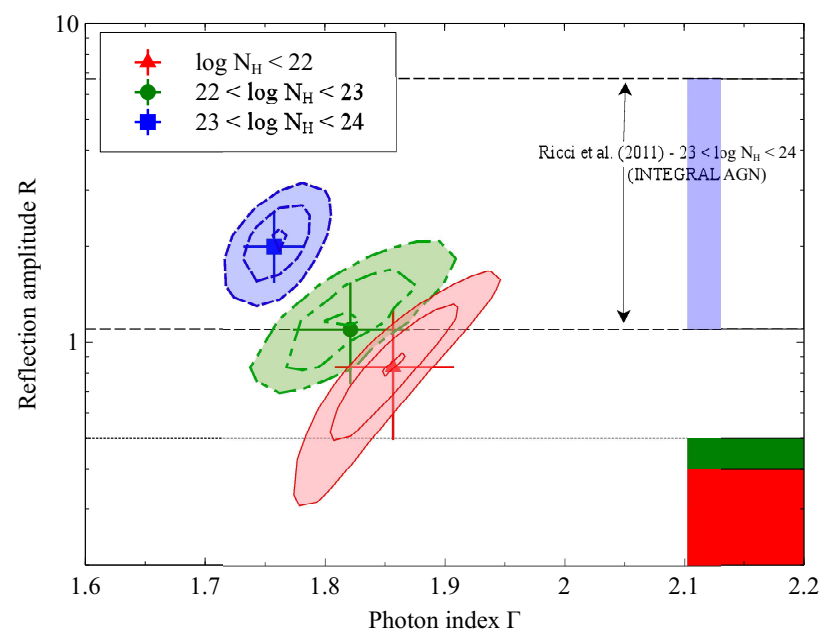

Fig. 3.- Contours for reflection amplitude $R$ against photon index $\Gamma$. Red solid line contours: 'unabsorbed' $\left(\log \mathrm{N}_{\mathrm{H}}<22\right)$ AGN; green dot-dashed contours: 'LOB' AGN $\left(22<\log \mathrm{N}_{\mathrm{H}}<23\right)$; blue dashed contours: 'MOB' AGN $\left(23<\log \mathrm{N}_{\mathrm{H}}<24\right)$. Contours represent a total change in $\chi^{2}$ of 10.0 from the minimal $\chi^{2}$ (number of degrees of freedom are 15, 13 and 15 for unabsorbed, LOB and MOB classes respectively). Colored shaded regions on the righthand side of the plot indicate the ranges of reflection parameters found for different absorption groups by Ricci et al. (2011). The position of these shaded areas does not reflect the photon index distribution from Ricci et al. (2011).

function with redshift, the total emission from sources at $z \sim 0$ is very similar to the sum over all redshifts from the XRB. Errors in the XRB normalization and Poisson fluctuations in our source numbers contribute uncertainties of $\sim 10 \%$ to the relative normalization.

This low-redshift analysis has the considerable benefit of good-quality data across the entire $0.4-200 \mathrm{keV}$ band, unavailable for much fainter higher redshift sources that make up the bulk of the XRB, allowing a direct decomposition into component source populations across a substantial part of the XRB spectral energy range. Most 


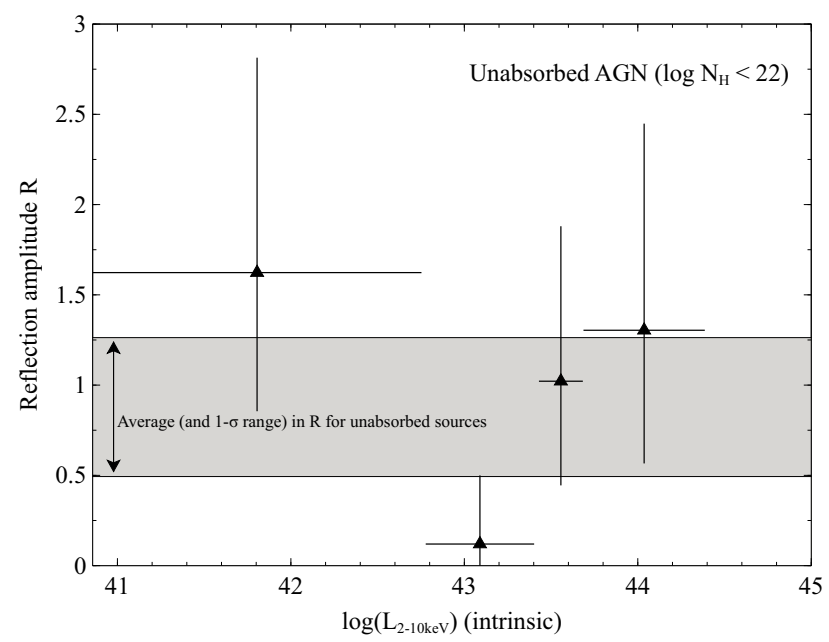

FIG. 4.- Reflection vs intrinsic $2-10 \mathrm{keV}$ luminosity for $\log \mathrm{N}_{\mathrm{H}}<$ 22 sources, stacked in different luminosity bins.

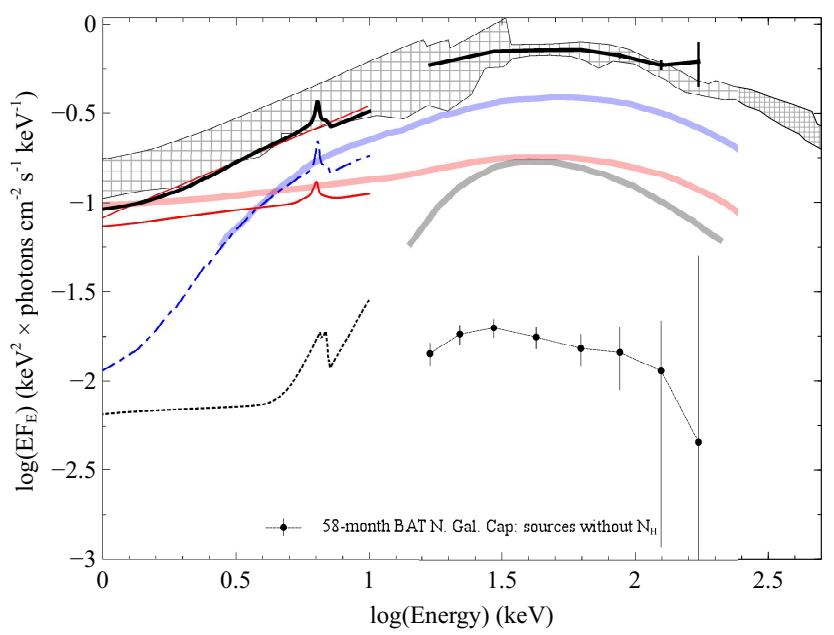

Fig. 5.- Key as for Fig. 1 Filled circles joined by dotted lines represent the contribution from five sources without sufficient counts at $0.4-10 \mathrm{keV}$ to determine their $N_{\mathrm{H}}$ values and contribution below $10 \mathrm{keV}$.

importantly, our analysis covers the peak emission at $\sim 30 \mathrm{keV}$. We employ a complete, relatively absorptionunbiased subsample drawn from a hard X-ray selected catalog.

Comparing the stacked spectra to the G07 synthesis model, below $10 \mathrm{keV}$ we find excellent agreement in the spectral shapes and the proportions of sources required to make up the emission. Above $10 \mathrm{keV}: 1$ ) the total emission has a slightly higher normalization than the XRB but within errors; 2) the hard excess or effective 'reflection' component for absorbed Compton-thin sources is higher than predicted by G07; and 3) the Comptonthick contribution is between $20-40 \%$ lower than the G07 model prediction, broadly consistent with the T09 model. Compton reflection, or an equivalent process, contributes to the hard excess particularly for $23<\log \mathrm{N}_{\mathrm{H}}<24$ sources, as found by Ricci et al. (2011) for INTEGRAL AGN. This does not seem to be due to an anti-correlation of luminosity with reflection, which is absent in our sample.

Our observed Compton-thick fraction is consistent with the G07 prediction at our survey flux limit (Fig. 17 of G07), even though it is not consistent with the overall flux- and luminosity-integrated Compton-thick fraction of the G07 model. However, we consistently compare our results with the full flux- and luminosity-integrated model synthesis results, since the key point here is that the stacked emission of a local, bright, but proportionately tiny fraction of the XRB surprisingly reproduces its shape, despite the flux limit. As such, these findings are not necessarily extendable to the entire XRB synthesis-model parameter space, but suggest interesting possibilites for future studies.

We have not included the contribution of blazars and other jet-dominated objects but they may be a significant contributor to the XRB; Draper \& Ballantyne (2009) find a $7 \%$ contribution in $2-10 \mathrm{keV}$ and $9 \%$ in $15-55 \mathrm{keV}$. However, blazars are only $3 \%$ of our $z<0.2$ sample, and are therefore not significant here.

The integrated spectrum at $z=0$ is surprisingly similar to that averaged over all cosmic time despite a known strong evolution in properties with redshift (Aird et al. 2010, Ueda et al. 2003). There is accumulating evidence now that the XRB shape is consistent with a smaller Compton-thick fraction (13\%) and higher reflection (notably $R=2.0$ for moderately absorbed $23<\log \mathrm{N}_{\mathrm{H}}<24$ sources), supporting the idea that such sources may be the main contributor to the XRB (Fabian et al. 1990; Gandhi et al. 2007), or that very complex absorption may play a role (Tatum et al. 2013). NuSTAR observations will be key for definitively answering these questions.

\section{ACKNOWLEDGEMENTS}

We thank the anonymous referee, Prof. R. Gilli and Dr. R. Krivonos for helpful suggestions which improved this work. We thank Dr. A. Akylas and Dr. A. Georgakakis for clarifying some of the results in Akvlas et al. (2012) and Prof. W. N. Brandt for useful discussions on the 58-month BAT catalog Northern Galactic Cap sample.

\section{REFERENCES}

Aird, J., Nandra, K., Laird, E. S., et al. 2010, MNRAS, 401, 2531

Aird, J., Coil, A. L., Moustakas, J., et al. 2012, ApJ, 746, 90

Ajello, M., Greiner, J., Sato, G., et al. 2008, ApJ, 689, 666

Akylas, A., Georgakakis, A., Georgantopoulos, I., Brightman, M. \& Nandra, K. 2012, A\&A, 546, A98

Antonucci, R. 1993, ARA\&A, 31, 473

Barger, A. J., Cowie, L. L., Mushotzky, R. F., et al. 2005, AJ, 129,578
Boldt, E. 1987, in IAU Symposium, Vol. 124, Observational Cosmology, ed. A. Hewitt, G. Burbidge, \& L. Z. Fang, 611-615

Burlon, D., Ajello, M., Greiner, J., et al. 2011, ApJ, 728, 58

Churazov, E., Sunyaev, R., Revnivtsev, M., et al. 2007, A\&A, 467,529

Delgado-Serrano, R., Hammer, F., Yang, Y. B., et al. 2010, A\&A, 509, A78

Draper, A. R., \& Ballantyne, D. R. 2009, ApJ, 707, 778 
Fabian, A. C., George, I. M., Miyoshi, S., \& Rees, M. J. 1990, MNRAS, 242, 14P

Frontera, F., Orlandini, M., Landi, R., et al. 2007, ApJ, 666, 86

Gandhi, P., Fabian, A. C., Suebsuwong, T., et al. 2007, MNRAS, 382,1005

Gilli, R., Comastri, A., \& Hasinger, G. 2007, A\&A, 463, 79

Iwasawa, K., \& Taniguchi, Y. 1993, ApJ, 413, L15

Lanzuisi, G., Civano, F., Elvis, M., et al. 2013, ArXiv e-prints

Malizia, A., Stephen, J. B., Bassani, L., et al. 2009, MNRAS, 399 944

Moretti, A., Pagani, C., Cusumano, G., et al. 2009, A\&A, 493, 501

Nandra, K., George, I. M., Mushotzky, R. F., Turner, T. J., \& Yaqoob, T. 1997, ApJ, 488, L91

Page, K. L., Reeves, J. N., O'Brien, P. T., \& Turner, M. J. L. 2005, MNRAS, 364, 195

Piconcelli, E., Jimenez-Bailón, E., Guainazzi, M., et al. 2005, A\&A, 432, 15

Ricci, C., Walter, R., Courvoisier, T. J.-L., \& Paltani, S. 2011, A\&A, 532, A102
Sazonov, S., Krivonos, R., Revnivtsev, M., Churazov, E., \& Sunyaev, R. 2008, A\&A, 482, 517

Sołtan, A. M. 2008, A\&A, 490, 1039

Tatum, M. M., Turner, T. J., Miller, L., \& Reeves, J. N. 2013, ApJ, 762, 80

Treister, E., Urry, C. M., \& Virani, S. 2009, ApJ, 696, 110

Türler, M., Chernyakova, M., Courvoisier, T. J.-L., et al. 2010, A\&A, 512, A49

Ueda, Y., Akiyama, M., Ohta, K., \& Miyaji, T. 2003, ApJ, 598, 886

Vasudevan, R. V., Brandt, W. N., Mushotzky, R. F., et al. 2013, ApJ, 763, 111

Williams, O. R., Turner, M. J. L., Stewart, G. C., et al. 1992 , ApJ, 389, 157

Worsley, M. A., Fabian, A. C., Bauer, F. E., et al. 2006, MNRAS, 368,1735 